\title{
Long-term histological comparison between near-infrared irradiated skin and scar tissues
}

This article was published in the following Dove Press journal:

Clinical, Cosmetic and Investigational Dermatology

24 November 2010

Number of times this article has been viewed

\author{
Yohei Tanaka ${ }^{1,2}$ \\ Kiyoshi Matsuo' \\ Shunsuke Yuzuriha' \\ 'Department of Plastic and \\ Reconstructive Surgery, Shinshu \\ University School of Medicine, \\ Matsumoto, Japan; ${ }^{2}$ Clinica Tanaka \\ Plastic and Reconstructive Surgery \\ and Anti-aging Center, Matsumoto, \\ Japan
}

Correspondence: Yohei Tanaka Department of Plastic and Reconstructive Surgery, Shinshu University School of Medicine, Asahi3-I-I, 390-862I

Matsumoto, Nagano, Japan

$\mathrm{Tel}+8 \mathrm{I}-263-37-2833$

Fax +8I-263-37-1920

Email yoheit@shinshu-u.ac.jp
Background and objective: Our previous histological studies indicated that near-infrared (NIR) irradiation stimulates collagen proliferation in rat and human skin for 3 months. High collagen density in the dermis and smoothing of the epidermis were observed in irradiated rat skin, and appeared to last up to 6 months. Epidermal smoothness in irradiated rat skin seems to resemble scarring. Here, we performed a long-term histological comparison between NIR (1100 to $1800 \mathrm{~nm}$ ) irradiated skin and scar tissues.

Materials and methods: Rat skin was irradiated using a NIR device. Scar tissues were harvested from wounded areas and were compared with irradiated skin. Histological changes up to 180 days post-treatment were evaluated with hematoxylin and eosin, Azan-Mallory staining, and collagen type I and III staining.

Results: In nonirradiated control skin, the dermis showed a low density of type I and III collagen, the surface of the epidermis was rough, and no significant changes were observed over time. In irradiated skin, both type I and III collagen increased significantly, and persisted up to 180 days. The density of type I collagen was significantly higher than that of type III collagen, whereas type I and III collagen of the control group did not differ significantly. Epidermis was thickened for 30 days, and epidermal smoothness persisted up to 180 days. In scar tissues, the density of type III collagen was higher than that of type I collagen. The number of fibroblasts remained high and the glial fibrils were dense until 180 days after injury compared with irradiated skin. Significant increases in both type I and III collagen and epidermal flattering persisted until 180 days.

Conclusions: NIR irradiation induced high collagen density in the dermis, resulting in long-term epidermal smoothness without scar formation. Results indicated that NIR irradiation provides safe, consistent, and long-term effects of skin rejuvenation.

Keywords: near-infrared irradiation, long-term effects, collagen, scar, skin rejuvenation

\section{Introduction}

Skin aging is a complex biological phenomenon that affects the different components of the skin, and consists of photoaging and intrinsic aging. ${ }^{1,2}$ Skin aging is commonly associated with skin wrinkling, sagging, and laxity. Skin laxity is a common complaint of patients who request skin rejuvenation. ${ }^{3}$ Many studies reported that a near-infrared (NIR) device with contact cooling is effective in the treatment of skin laxity. ${ }^{3-8}$ Nonablative lasers and radiofrequency devices work by thermally stimulating dermal collagen remodeling. ${ }^{9}$ Thermal damage, including NIR irradiation, denatures the collagen and encourages the generation of new collagen, resulting in tighter skin. ${ }^{5,6}$ NIR irradiation produces clearly measurable elevations in markers of 
collagen production. ${ }^{10}$ NIR irradiation increases the amounts of collagen in human dermal fibroblasts, and can result in clinical improvement of skin texture. ${ }^{11}$

We previously reported that NIR irradiation induces an injury response in the skin and subcutaneous tissues. ${ }^{12,13}$ NIR irradiation also induces preferential production of type I collagen without a long-term increase in type III collagen production for 3 months following NIR irradiation in rat skin. ${ }^{12}$ High collagen density in the dermis and smoothing of the epidermis were observed in irradiated skin at postirradiation day 30 (P30), ${ }^{12}$ and appeared to last up to P180. Smoothness of the epidermis for such a long period may be thought of as scarring. However, irradiated skin appeared different from scars.

We hypothesized that NIR irradiation induces long-term high collagen density in the dermis and smoothing of the epidermis without scar formation. To verify this hypothesis, we examined the histological changes over time after NIR irradiation and compared the results with those of scar tissues.

\section{Materials and methods} Animal preparations

This study was approved by Shinshu University Institutional Review Board for Animal Study. Thirty male Wistar rats (Rattus norvegicus albino) were prepared for this study. Animals were housed in a temperature-controlled environment under a 12-hour light/dark cycle with free access to water and standard rat chow. Before treatments and tissue sampling, the rats were anesthetized using intraperitoneal injection of $50 \mathrm{mg} / \mathrm{kg}$ pentobarbitone. Tissue samples were obtained from a rectangular fragment of treated skin and subcutaneous tissue measuring approximately $30 \times 20 \mathrm{~mm}$ in the median region of the back. Scar tissue samples were harvested from the intentionally wounded skin measuring approximately $20 \times 10 \mathrm{~mm}$ in the back. National and international principles of laboratory animal care were followed throughout this study.

\section{Near-infrared irradiation}

Animals were divided into 2 groups: control group $(n=5)$ and irradiated group $(n=25)$. The irradiated group received treatments at $40 \mathrm{~J} / \mathrm{cm}^{2}$, while the control group received no irradiation. Rats in the irradiated group received 3 treatments on days 0,7 , and 14. Treatments consisted of 2 passes of NIR irradiation. All groups were monitored and measured simultaneously.

NIR irradiation was performed with a broadband NIR source (Titan; Cutera, Brisbane, CA). The NIR device emits a spectrum of NIR between 1100 and $1800 \mathrm{~nm}$, with filtering of wavelengths between 1400 and $1500 \mathrm{~nm}$ that are strongly absorbed by fluid and hemoglobin. Surface temperature was regulated with an integrated temperature-controlled sapphire window to prevent epidermal burns during irradiation. ${ }^{4-6,8}$ This specific wavelength and cooling system enabled deeper penetration of NIR and sufficient NIR delivery without any pain or epidermal burns.

Fluence ranges for the study were determined based on clinical experience with the device and our previous report. We reported that NIR irradiation at a dose of $20 \mathrm{~J} / \mathrm{cm}^{2}$ is sufficiently high to induce histological changes in the epidermis, but that higher energies have a greater response, and are preferable for dermal effects, such as skin tightening. ${ }^{12}$ Therefore, the irradiated group was treated at a dose of $40 \mathrm{~J} / \mathrm{cm}^{2}$ in the present study.

\section{Histological investigation}

Over 60 skin samples were obtained for microscopic investigation. Biopsies were taken on postirradiation days $0,7,30,45$, and 180 (P0, P7, P30, P45, and P180, respectively). The specimens were fixed in $20 \%$ neutral buffered formalin and processed for paraffin embedding. Specimens were then serially sectioned in the sagittal plane (3-4 $\mu \mathrm{m}$ thick) and evaluated with hematoxylin and eosin, Azan-Mallory staining, and immunohistochemical staining. Collagen staining was performed with purified rabbit anti-rat collagen type I and III polyclonal antibodies (Novotec, Lyon, France) at a dilution of 1:500. The sections were examined and photographed with a digital camera system and processed with Adobe Photoshop (Adobe, San Jose, CA).

To calculate the area of the stained regions in the dermis, an optimized color threshold was applied to each image to distinguish between the stained areas and background. The proportion of the selected color was calculated as a percentage of the total area and used as a measure of collagen density. Collagen synthesis was scanned and quantified in 5 representative fields per section. The scores of the 5 fields were then averaged to obtain a final score for each section. Two independent physicians performed the histology interpretation and the image analysis.

\section{Statistical analysis}

Data presented are means $\pm \mathrm{SD}$. We performed statistical analyses with Stat View system (SAS Institute Inc.). The differences between groups at each time point were examined for statistical significance with the Mann-Whitney 
$U$-test test. $P<0.05$ was considered to indicate statistical significance.

\section{Results}

Histological investigation exhibited changes over time as shown in Figures 1 to 3. Results of measurements of types I and III collagen are shown Figure 4.

In the control group, the layer of keratin was very rough and uneven (Figures 1 and 3). The dermis showed a low density of type I and III collagen (Figure 1). No significant changes were observed in the epidermis or dermis in the control group throughout the present study.

In irradiated skin, smoothing and thickening of the epidermis, hyperplasia of fibroblasts, lymphocytes, and small blood vessels in a wide area of the dermis were detected

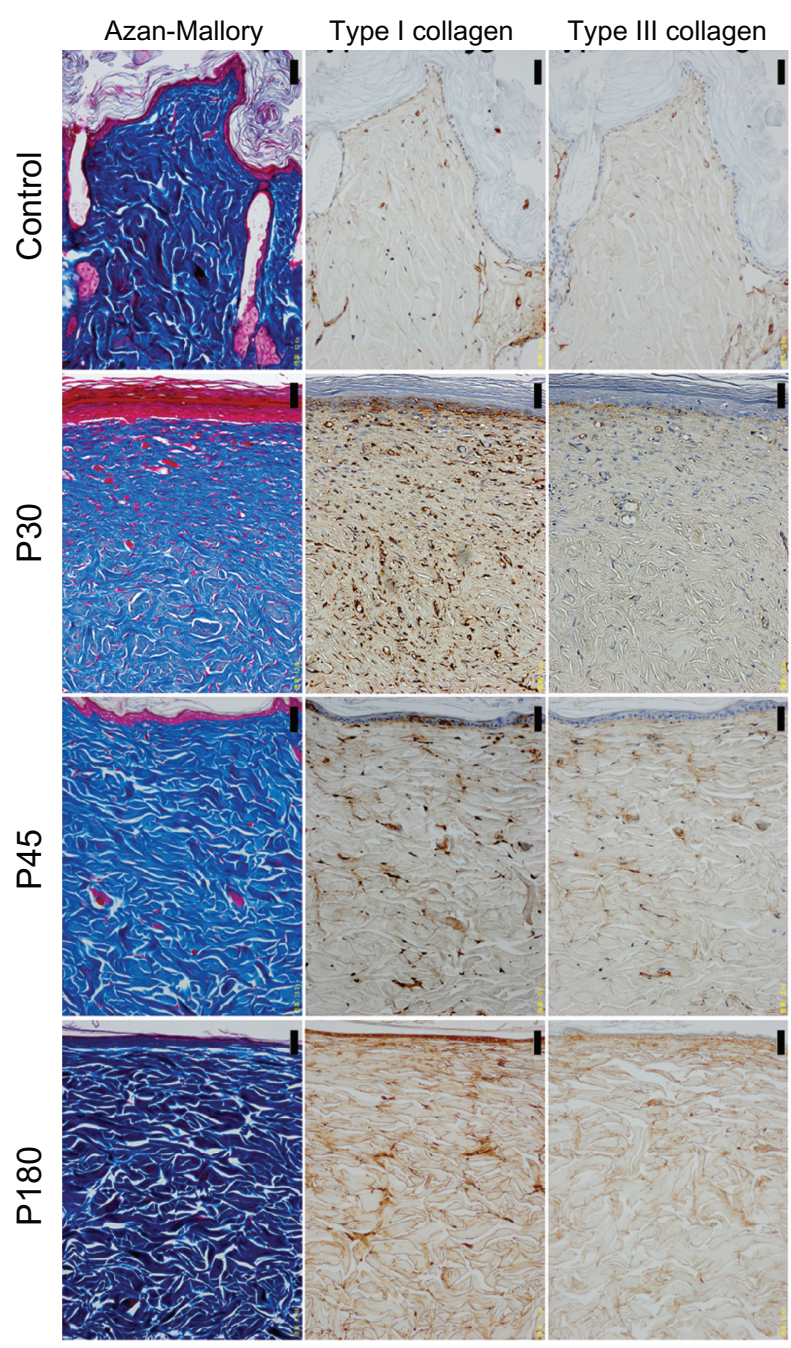

Figure I The histology of control and near-infrared (NIR) irradiated skin $(\times 200$ magnification). The left column shows Azan-Mallory staining, the middle column shows type I collagen staining, and the right column shows type III collagen staining. Images from top to bottom show control, postirradiation day 30 (P30), postirradiation day 45 (P45), postirradiation day 180 (PI80). Scale bar $=50.0 \mu \mathrm{m}$. in biopsies taken on P0 and P7 (Figure 3). The dermis was edematous up to P7. Smoothing of the epidermis and hyperplasia of fibroblasts with large nuclei in a wide area of the dermis were also observed in biopsies taken on P30. Further smoothing of the epidermis and bunch-shaped increases in glial fibrils were identified with Azan-Mallory staining on P45. The number of fibroblasts decreased and most of their nuclei became spindle-shaped on P45, which approached those in the controls. Both type I and III collagen increased compared with the control group. High collagen density in the dermis and smoothness of the epidermis were still observed on P180.

The histological appearance of irradiated skin appeared different from that of scar tissues. In scar tissues, epidermal atrophy, follicular plugging, fibroplasia, and

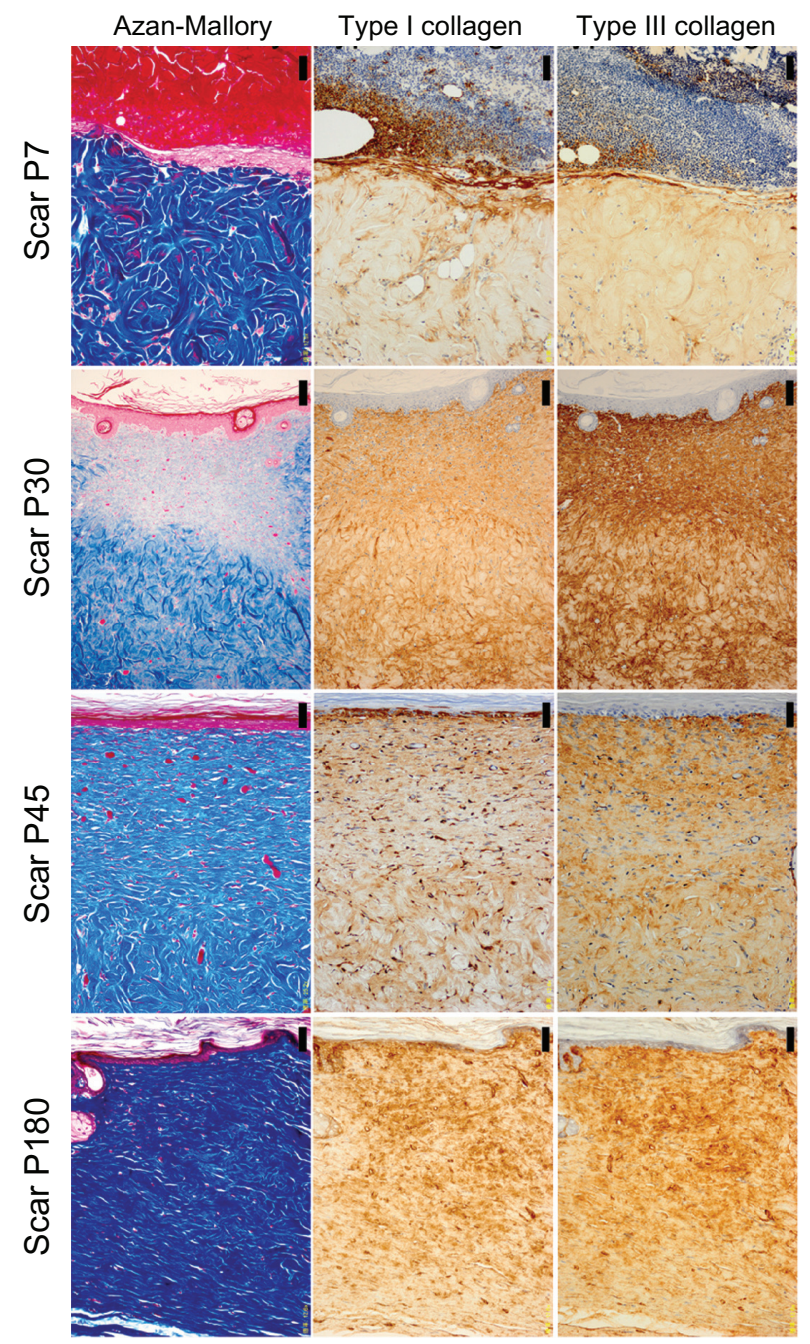

Figure 2 The histology of scar tissues ( $\times 200$ magnification). The left column shows Azan-Mallory Staining, the middle column shows type I collagen staining, and the right column shows type III collagen staining. Images from top to bottom show postinjury day 7 (P7), postinjury day 30 (P30), postinjury day 45 (P45), postinjury day 180 (PI80). Scale bar $=50.0 \mu \mathrm{m}$. 


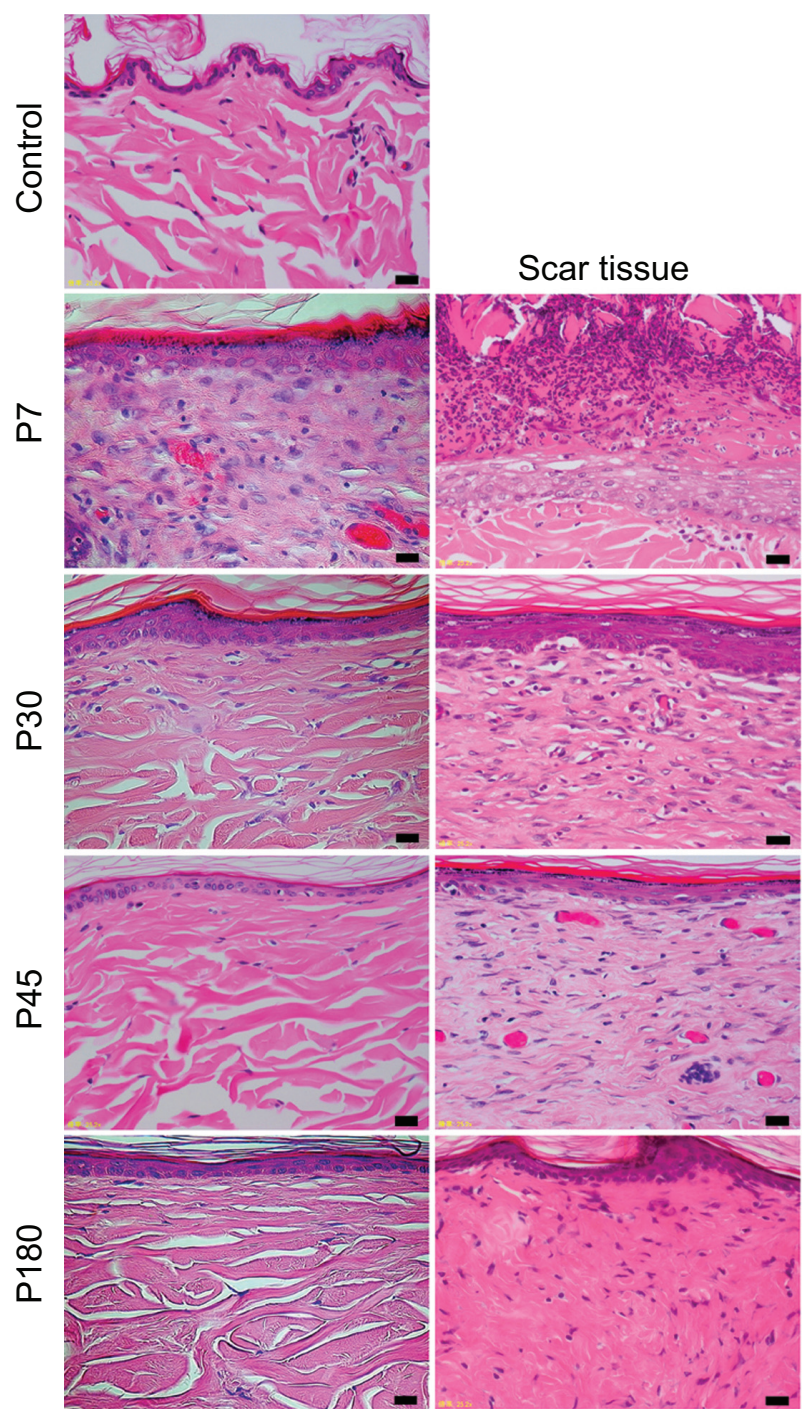

Figure 3 The histology of control, near-infrared (NIR) irradiated tissues, and scar tissues with hematoxylin and eosin staining ( $\times 400$ magnification). The left column shows control skin and NIR irradiated skin, and the right column shows scar tissues. Images from top to bottom show control, P7, P30, P45, and PI80. Scale bar $=20.0 \mu \mathrm{m}$

angioplasia with dense collagen bundles were identified (Figure 2 and 3). The number of fibroblasts remained high and the glial fibrils were dense until P180 after injury compared with irradiated skin (Figure 3). Both type I and III collagen increased compared with the control group, and the density of type III collagen appeared higher than that of type I collagen. High collagen density in the dermis and flattering of the epidermis were also still observed until P180 in scar tissues.

Investigation of the percentages of collagen density in irradiated skin revealed that significant increases in both type I and III collagen persisted up to P180 compared with the control group $(P<0.05)$. The density of type I collagen was significantly higher than that of type III collagen in irradiated skin at P30, P45, and P180 $(P<0.05)$, whereas type I and III collagen of the control group did not differ significantly $(P=0.251)$ (Figure 4$)$. In scar tissues, the density of type III collagen was higher than that of type I collagen (Figures 2 and 4). Significant increases in both type I and III collagen persisted until P180 compared with the control group $(P<0.05)$. The density of type III collagen was significantly higher than that of type I collagen at $\mathrm{P} 30(P<0.05)$, whereas a significant difference was not observed between type I and III collagen at P7, P45, and P180 ( $P=0.754,0.0758,0.251$, respectively) (Figure 4).

\section{Discussion}

The safety and efficacy of modern lasers are attributable to the work of Anderson and Parrish. ${ }^{14}$ Utilizing a wavelength range of 1100 to $1800 \mathrm{~nm}$ and contact cooling, this NIR device is able to target water without targeting melanin or hemoglobin, allowing safe treatment of the deep dermis without risk to the basement membrane.

Some authors reported that clinical improvement following dermabrasion of photo-aged skin is correlated with synthesis of collagen I. $^{15,16}$ Orringer et al reported that both type I and type III collagen are important in dermal proliferation after ablative laser resurfacing. ${ }^{17}$ We previously reported that NIR irradiation induces preferential production of type I collagen without a long-term increase in type III collagen 3 months following NIR irradiation in rat skin. ${ }^{12}$ Type I collagen stimulation persists for 90 days in rat tissue, while type III collagen stimulation shows no significant difference from controls by $\mathrm{P} 45 .{ }^{12}$ However, in the present study, the density of type I collagen remained high compared with type III collagen, and high collagen density in the dermis and smoothness of the epidermis were still observed up to 180 days after NIR irradiation. Although we performed evaluations for 180 days in the present study, these effects may last longer.

Many different NIR devices and lasers were used in previous studies, typically utilizing wavelengths between 750 and $3000 \mathrm{~nm}$. Wavelength selection directly influences target selection and penetration depth. Wavelengths below $1100 \mathrm{~nm}$ are absorbed preferentially by melanin in the superficial layers of the skin. Wavelengths between 1400 and $1500 \mathrm{~nm}$ and above $1850 \mathrm{~nm}$ are absorbed strongly by water, resulting in heating of the superficial layers of the skin, which can lead to painful sensations and burns. ${ }^{18}$ In this study, we used a NIR device that emits a spectrum of NIR from 1100 to $1800 \mathrm{~nm}$, with filtering of wavelengths between 1400 and $1500 \mathrm{~nm}$ that are strongly absorbed by fluid and hemoglobin. Filtering out these wavelengths below $1100 \mathrm{~nm}$, 


\section{Control and NIR irradiated skin}

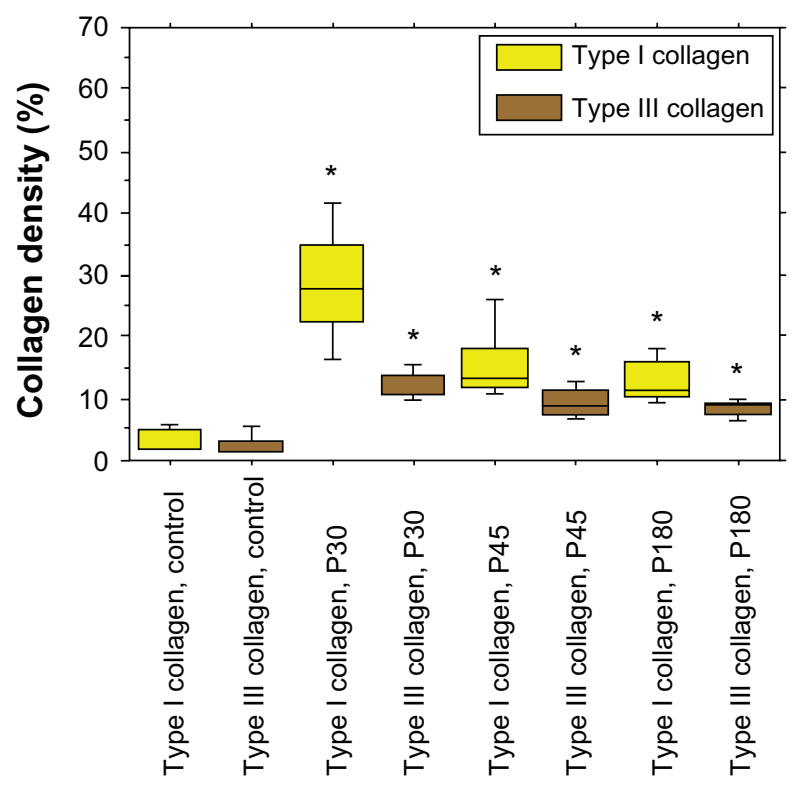

\section{Scar tissues}

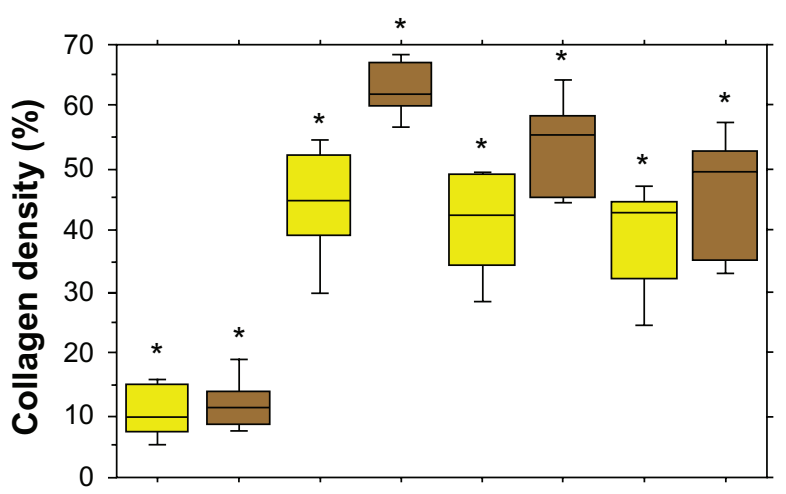

\begin{tabular}{|c|c|c|c|c|c|c|}
\hline & $\hat{\alpha}$ & ஓి & ஜ & $\stackrel{10}{\mathcal{Y}}$ & $\stackrel{10}{\square}$ & $\stackrel{\infty}{\infty}$ \\
\hline $\begin{array}{l}\frac{\tilde{\Phi}}{\Phi} \\
\frac{\mathbb{D}}{\bar{D}} \\
\overline{\bar{O}}\end{array}$ & $\begin{array}{l}\frac{5}{0} \\
\stackrel{0}{0} \\
\frac{\mathbb{0}}{\overline{0}} \\
\underline{0}\end{array}$ & $\begin{array}{l}\frac{c}{\Phi} \\
\text { D্ঠ } \\
\frac{\bar{D}}{0}\end{array}$ & 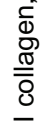 & 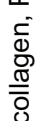 & 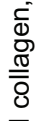 & $\begin{array}{l}\frac{1}{\Phi} \\
\frac{\delta}{D} \\
\frac{\mathbb{D}}{\bar{O}}\end{array}$ \\
\hline & $\equiv$ & - & $\equiv$ & - & $\equiv$ & - \\
\hline 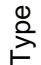 & 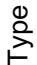 & 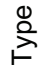 & 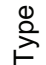 & 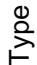 & 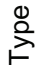 & 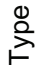 \\
\hline
\end{tabular}

Figure 4 The percentages of type I collagen (yellow), and type III collagen (brown). Left: the results of control, and near-infrared (NIR) irradiated skin, right: the results of scar tissues.

Note: Asterisks indicate statistical significance $(P<0.05)$ compared to the controls.

around $1450 \mathrm{~nm}$ and above $1850 \mathrm{~nm}$ enables delivery of light energy or NIR safely to the deeper tissues. ${ }^{19}$ Without filtering, too much energy is absorbed in the superficial layers of the skin, limiting NIR delivery to deeper tissue. Further, contact cooling helps further prevent severe increases in the surface temperature, ${ }^{6}$ to allow delivery of NIR safely and efficiently to the deeper tissues. ${ }^{5,19}$ This specific wavelength and cooling system enable NIR to penetrate to deeper tissues without any pain or epidermal burns.

The energy produced by the Titan infrared handpiece penetrates into the skin and is absorbed primarily by water within the dermis, resulting in heating throughout the skin., ${ }^{4,5}$ This absorption increases the temperature, causing the release of inflammatory chemical mediators that stimulate the collagen healing process. These inflammatory mediators released from vascular endothelial cells induce new dermal collagen production by fibroblasts. ${ }^{20}$ Dermal injury after a single NIR irradiation treatment indicates that NIR irradiation affects various aspects of the healing process, including the degree of inflammation, formation and organization of the collagen, neovascularization, and epithelization. ${ }^{21}$

To evaluate the effects of NIR irradiation, we investigated biopsies taken on each of P0, P7, P30, P45, and P180. Hyperplasia of fibroblasts, lymphocytes, and small blood vessels, and edematous changes in a wide area of the dermis were observed on $\mathrm{P} 0$ and $\mathrm{P} 7$. These findings suggest that the period around $\mathrm{P} 0$ and $\mathrm{P} 7$ represents the inflammation stage in rat tissue. Demir reported that the breaking strength used for biomechanical evaluation of wounds in the maturation phase increases significantly after the third week of healing in rats. ${ }^{22}$ In the present study and our previous investigations, hyperplasia of fibroblasts and small blood vessels in a wide area of the dermis were observed, and the nuclei of fibroblasts were still large on P30, suggesting that P30 is within the proliferation stage.

On P45, the number of fibroblasts decreased and most of their nuclei became spindle shaped, and increases in glial fibrils were identified with Azan-Mallory staining, although the glial fibrils were thin on P30. These observations suggest that the proliferation stage lasts for 1 month, and the maturation stage begins at day 45 in rat tissue. ${ }^{12,13}$ In human tissue, the proliferation stage is thought to last for about 1 to 3 months, and the maturation stage begins at around 2 to 3 months. ${ }^{13}$ Thus, the human healing period is about 2 to 3 times longer than that in rats. ${ }^{13}$ The effects of NIR irradiation on type I collagen were estimated to fade gradually over 3 to 4 months in rat tissue, extrapolating to roughly 1 year in human tissue. ${ }^{12,13}$

These findings are consistent with those of the previous report that collagen remodeling in humans continues for 6 to 12 months after traumatic stimulation of fibroblasts. ${ }^{11}$ High collagen density and epidermal smoothness for such a long period may be thought of as scarring. However, histological appearance of irradiated skin appeared different from that 
of scar tissues. Hypertrophic scars are characterized by epidermal atrophy, follicular plugging, fibroplasia, and angioplasia with dense collagen bundles replacing the dermis. ${ }^{23}$ In the present study, all of these findings were identified in scar tissues, whereas follicular plugging, fibroplasia, and angioplasia with dense collagen bundles were not identified in irradiated skin.

Furthermore, our previous study and the present histological investigation suggest that NIR irradiation does not induce scar formation because of the very small amounts of type III collagen observed on P45, P90, and P180, which is usually seen in scar tissues. An increase in type III collagen was observed in scar tissues, ${ }^{24,25}$ and the same results were observed in the present study. The skin of elderly subjects shows an increase in type III collagen in normally sunprotected skin, and a marked increase in type III collagen in sun-exposed sites compared with sun-protected sites. ${ }^{25,26}$ Type I collagen appears to be a softer form of collagen, while type III collagen is more rigid and is found frequently in scar tissue. ${ }^{12}$ Therefore, it is preferable to stimulate type I collagen production for skin rejuvenation. As the density of type I collagen remained high compared with type III collagen and smoothness of the epidermis was still observed up to 180 days after NIR irradiation, the results support the conclusion that this NIR irradiation method can induce safe and long-term skin rejuvenation effects. High collagen density in the dermis may support long-term smoothness of the epidermis, and further investigations in this area are therefore warranted.

It should be noted that this was a preliminary study based on animal experiments in a limited number of biopsies. Future studies could include additional assays, such as qPCR or western blotting to obtain more quantitative evidence. Also, functional measurements such as the elastic modulus of the treated skin would be highly informative. Other areas of possible investigation include evaluating dose effects and other laser parameters.

In conclusion, the results of the present study indicated that a specialized wavelength of NIR 1100 to $1800 \mathrm{~nm}$ with filtering of wavelengths between 1400 and $1500 \mathrm{~nm}$, induces long-term high collagen density in the dermis, resulting in long-term smoothness of the epidermis without scar formation. Clinical application of the results from our experiments indicate that this NIR irradiation method using specialized wavelengths does not result in scar, and provides safe, consistent, and long-term skin rejuvenation effects. This is preferential for cosmesis in patients wishing improvement of laxity and wrinkles with smoother, more youthful skin.

\section{Acknowledgments}

We thank Ikuo Matsuyama for histological staining, and members of Cutera Inc. for providing technical information on the NIR device and helpful comments.

\section{Disclosure}

The authors report no conflicts of interest.

\section{References}

1. Kligman AM, Lavker RM. Cutaneous aging: the differences between intrinsic aging and photoaging. J Cut Aging Cosmet Dermatol. 1988;1:5-12.

2. Uitto J. The role of elastin and collagen in cutaneous aging: intrinsic aging versus photoexposure. J Drugs Dermatol. 2008;7:12-16.

3. Chan HH, Yu CS, Shek S, et al. A prospective, split face, single-blinded study looking at the use of an infrared device with contact cooling in the treatment of skin laxity in Asians. Lasers Surg Med. 2008;40: $146-152$.

4. Esparza JR. Near painless, nonablative, immediate skin contraction induced by low-fluence irradiation with new infrared device: a report of 25 patients. Dermatol Surg. 2006;32:601-610.

5. Zelickson B, Ross V, Kist D, et al. Ultrastructural effects of an infrared handpiece on forehead and abdominal skin. Dermatol Surg. 2006;32: 897-901.

6. Goldberg DJ, Hussain M, Fazeli A, et al. Treatment of skin laxity of the lower face and neck in older individuals with a broad-spectrum infrared light device. J Cosmet Laser Ther. 2007;9:35-40.

7. Chua SH, Ang P, Khoo LSW, Goh CL. Nonablative infrared skin tightening in type IV to V Asian skin: a prospective clinical study. Dermatol Surg. 2007;33:146-151.

8. Alexiades-Armenakas M. Assessment of the mobile delivery of infrared light (1100-1800 nm) for the treatment of facial and neck skin laxity. J Drugs Dermatol. 2009;3:221-226.

9. Lipper GM, Perez M. Nonabrative acne scar reduction after a series of treatments with a short-pulsed 1064-nm neodymium: YAG laser. Dermatol Surg. 2006;32:998-1006.

10. Orringer JS, Voorhees JJ, Hamilton T, et al. Dermal matrix remodeling after nonablative laser therapy. JAm Acad Dermatol. 2005;53: 775-782.

11. Lee JH, Roh MR, Lee KH. Effects of infrared radiation on skin photo-aging and pigmentation. Yonsei Medical Journal. 2006;47: 485-490.

12. Tanaka Y, Matsuo K, Yuzuriha S, Shinohara H. Differential long-term stimulation of type I versus type III collagen after infrared irradiation. Dermatol Surg. 2009;35:1099-1104.

13. Tanaka Y, Matsuo K, Yuzuriha S. Long-term evaluation of collagen and elastin following infrared (1100 to $1800 \mathrm{~nm})$ irradiation. J Drugs Dermatol. 2009;8:708-712.

14. Anderson RR, Parrish JA. Selective photothermolysis: precise microsurgery by selective absorption of pulsed radiation. Science. 1983;220:524-527.

15. Nelson BR, Majmudar G, Griffith CEM, et al. Clinical improvement following dermabrasion of photo-aged skin correlates with synthesis of collagen I. Arch Dermatol. 1994;130:1136-1142.

16. Griffiths CEM, Russman AN, Majmudar G, et al. Reduced collagen I in photoaged human is repaired by retinoic acid. N Engl J Med. 1993; 329:530-535.

17. Orringer JS, Kang S, Johnson TM, et al. Connective tissue remodeling induced by carbon dioxide laser resurfacing of photodamaged human skin. Arch Dermatol. 2004;140:1326-1332.

18. Kelleher DK, Thews O, Rzeznik J, Scherz A, Salomon Y, Vaupel P. Hot topic. Water-filtered infrared-A radiation: a novel technique for localized hyperthermia in combination with bacteriochlorophyll-based photodynamic therapy. Int J Hyperthermia. 1999;15:467-474. 
19. Davenport SA, Gollnick DA, Levernier M, Spooner GJR. Method and system for treatment of post-partum abdominal skin redundancy or laxity. United States Patent 20060052847. Available at; http://www. freepatentsonline.com/y2006/0052847.html

20. Lee MWC. Combination 532-nm and 1064-nm lasers for noninvasive skin rejuvenation and toning. Arch Dermatol. 2003;139:1265-1276.

21. Rezende SB, Ribeiro MS, Nunez SC, et al. Effects of asingle near-infrared laser treatment on cutaneous wound healing: Biometrical and histological study in rats. J Photochem Photobiol B: Biol. 2007; 87:145-153.

22. Demir H, Balay H, Kirnap M. A comparative study of the effects of electrical stimulation and laser treatment on experimental wound healing in rats. J Rehabil Res Dev. 2004;41:147-154.
23. Avram MM, Tope WD, Yu T, Szachowicz E, Nelson JS. Hypertrophic scarring of the neck following ablative fractional carbon dioxide laser resurfacing. Lasers Surg Med. 2009;41:185-188.

24. Bailey AJ, Bazin S, Sims TJ, et al. Characterization of the collagen of human hypertrophic and normal scars. Biochim Biophys Acta. 1975; 405:412-421.

25. Stephen R, Plastow B.Sc, John A, et al. Early changes in dermal collagen of mice exposed to chronic UVB irradiation and the effects of a UVB sunscreen. J Invest Dermatol. 1988;91:590-592.

26. Lovell CR, Smolenski KA, Duance VC, et al. Type I and type III collagen content and fibre distribution in normal human skin during ageing. Br J Dematol. 1987;117:419-428.

\section{Publish your work in this journal}

Clinical, Cosmetic and Investigational Dermatology is an international, peer-reviewed, open access, online journal that focuses on the latest clinical and experimental research in all aspects of skin disease and cosmetic interventions. All areas of dermatology will be covered; contributions will be welcomed from all clinicians and basic science researchers globally. This journal is indexed on CAS. The manuscript management system is completely online and includes a very quick and fair peer-review system, which is all easy to use. Visit http://www.dovepress.com/testimonials.php to read real quotes from published authors.

Submit your manuscript here: http://www.dovepress.com/clinical-cosmetic-and-investigational-dermatology-journal 